\title{
Beamtest of Non-irradiated and Irradiated ATLAS SCT Microstrip Modules at KEK
}

\author{
Y. Unno, T. Matuo, T. Hashizaki, T. Akimoto, J. Bernabeu, Z. Dolezal, L. Eklund, K. Hara, Y. Ikegami, Y. Iwata, \\ Y. Kato, C. Ketterer, H. Kobayashi, T. Kohriki, T. Kondo, T. Koshino, J. Ludwig, T. Masuda, G. Moorhead, \\ I. Nakano, K. Norimatsu, T. Ohsugi, K. Runge, S. Shinma, R. Takashima, R. Tanaka, N. Tanimoto, S. Terada, \\ N. Ujiie, M. Vos, K. Yamanaka, and T. Yamashita
}

\begin{abstract}
Non-irradiated and irradiated ATLAS SCT barrel and endcap modules have been beamtested with $4 \mathrm{GeV} / \mathrm{c}$ pions. Pulse shapes confirmed the peaking time of the amplifier to be $22 \mathrm{~ns}$ with slight deterioration in the irradiated modules. Median charges saturated around $3.8 \mathrm{fC}$ both in the non-irradiated and irradiated modules. Signal/Noise ratios, using the noise estimates from the in-situ calibration, were $>16$ in the non-irradiated $(>150 \mathrm{~V})$, and $>10$ in the irradiated $(>300 \mathrm{~V})$ barrel modules. No excess common-mode noise was observed.
\end{abstract}

\section{INTRODUCTION}

In order to investigate the origin of the mass of elementary particles and search for new physics, a new accelerator, LHC (Large Hadron Collider), is being built at CERN which will accelerate and collide protons at the centre of mass energy of $14 \mathrm{TeV}$, with a collision luminosity of $10^{34} \mathrm{~cm}^{-2} \mathrm{sec}^{-1}$ and a beam crossing interval of $25 \mathrm{~ns}$. In one of the LHC experiments, ATLAS, the detection and the measurement of momentum of charged particles will be made in the inner detector, consisting of pixel (PIXEL), silicon microstrip (SCT), and transition-radiation (TRT) detectors, inside a 2 Tesla solenoid magnet of a diameter of $2 \mathrm{~m} \mathrm{[1].}$

The SCT provides high-precision tracking information with

Manuscript received Nov. 23, 2001. This work was supported in part by the Grant-in-aid for Scientific Research of Japan Ministry of Education, Science, and Technology, Australian Research Council and Department of Industry, Science and Resources, German Ministry for Education and Research (BMBF), and Valencia University and the Spanish research council CSIC.

Y. Unno, T. Kohriki, T. Kondo, S. Terada, N. Ujiie are with the Institute of Particle and Nuclear Studies, High Energy Accelerator Research Organization (KEK), Tsukuba 305-0801, Japan

T. Matuo, T. Hashizaki, T. Koshino, I. Nakano, K. Norimatsu, R. Tanaka, N. Tanimoto, T. Yamashita are with the Physics department, Okayama University, Okayama 700-8530, Japan

C. Ketterer, J. Ludwig, K. Runge are with the Department of Physics, Albert-Ludwig University of Freiburg, D-79104 Freiburg, Germany

Z. Dolezal is with the Institute of Particle and Nuclear Physics, Charles

University, CZ-180 00 Prague 8, Czech Republic

Y. Iwata, T. Masuda, T. Ohsugi, K. Yamanaka are with the Physics department, Hiroshima University, Higashi-Hiroshima 739-8526, Japan

$\mathrm{R}$. Takashima is with the Education department, Kyoto University of Education, Kyoto 612-0863, Japan

G. Moorhead is with the School of Physics, University of Melbourne, Parkville, Victoria 3052, Australia

T. Akimoto, K. Hara, Y. Kato, H. Kobayashi, S. Shinma are with the Institute of Physics, University of Tsukuba, Tsukuba 305-8571, Japan

L. Eklund is with the Department of Radiation Science, Uppsala University, S-75121 Uppsala, Sweden

J. Bernabeu, M. Vos are with the Instituto de Fisica Corpuscular, Univ. Valencia/CSIC, E-46071, Valencia, Spain a position resolution of $23 \mu \mathrm{m}$ per sensing plane. It comprises a central barrel section of 4 cylinders and two endcap sections of 9 disks each. The cylinders or disks have two sensing planes per layer, with total areas of silicon of 34 and $27 \mathrm{~m}^{2}$ in the barrel and the endcap sections, respectively. The silicon microstrip sensors will receive a cumulative fluence of $2 \times 10^{14}$ $1 \mathrm{MeV}$-neutron-equivalent particles/ $\mathrm{cm}^{2}$ over 10 years of operation at the inner-most radius of $30 \mathrm{~cm}$ from the collision axis.

The silicon planes are implemented in detector units called "modules" made of silicon microstrip sensors and fast-shaping, on-off discriminating readout electronics, combined with electrical, mechanical, and thermal structures [2]. After intensive development, a number of realistic SCT modules, fully equipped with SCT-specified components, have become available, of which a significant number have been irradiated to the full fluence of particles.

A number of beamtests to study individual components have previously been carried out at KEK using pion beam of $4 \mathrm{GeV} / \mathrm{c}$ at the $12 \mathrm{GeV}$ proton synchrotron [3]. Recently, two beamtests were carried out of fully equipped SCT modules [4]. These were the first beamtests of realistic SCT modules.

\section{SCT SILICON MICROSTRIP MODULES}

\section{A. ATLAS SCT silicon microstrip sensor}

After intensive studies of the radiation damage in semiconductors and following the development of radiation-tolerant designs, ATLAS SCT has chosen a type of silicon microstrip sensors with p-implant strips in n-bulk silicon wafers, so-called p-in-n sensors. Several geometries of otherwise similar detector technology have been implemented; one, essentially square, is used for the barrel modules, and five different wedge shapes used for the endcap modules. The sensors are designed to have a mean strip pitch of $80 \mu \mathrm{m}$ at the centre of a module. The parameters of the barrel and the endcap sensors used in the beamtest are summarized in Table I [5].

\section{B. Frontend readout ASIC}

The signals from the strips are read out by frontend circuits with high gain $(50 \mathrm{mV} / \mathrm{fC})$, unipolar fast shaping (20 ns peaking time), on-chip discrimination (binary readout), a digital 
TABLE I

PARAMETERS OF THE ATLAS SCT P-IN-N SILICON MICROSTRIP SENSORS

\begin{tabular}{c|c|c}
\hline Module & Barrel & Endcap \\
\hline Sensor type & p-in-n, AC-coupled, Single-sided \\
\hline Thickness & square $285 \pm 15 \mu \mathrm{m}$ \\
\hline Shape & $63.6 \mathrm{~mm} \times 64.0 \mathrm{~mm}$ & $\begin{array}{c}71.8 / 64.7 \mathrm{~mm} \times 57.5 \mathrm{~mm} \\
64.6 / 56.5 \mathrm{~mm} \times 65.5 \mathrm{~mm}\end{array}$ \\
\hline $\begin{array}{c}\text { Size (outer) } \\
\text { (width } \times \text { length) }\end{array}$ & n-type, high resistivity (about $4 \mathrm{k} \Omega \mathrm{cm})$ \\
\hline Bulk & \multicolumn{2}{|c}{$\mathrm{p}^{+}$} \\
\hline Strip type & $80 \mu \mathrm{m}$ & $85.95,77.45 \mu \mathrm{m}$ \\
\hline Strip pitch & $62 \mathrm{~mm}$ & $55.5,63.5 \mathrm{~mm}$ \\
\hline Strip length & \multicolumn{2}{|c}{$16 \mu \mathrm{m},(22 \mu \mathrm{m}: \mathrm{Al} \mathrm{metal)}$} \\
\hline Strip width & \multicolumn{2}{|c}{768 readout +2 shaping } \\
\hline Number of strips & \multicolumn{2}{c}{ SiO2 + SiliconNitride, $>20 \mathrm{pF} / \mathrm{cm}$} \\
\hline Strip AC coupling & \multicolumn{2}{c}{}
\end{tabular}

pipeline for the trigger decision delay of $3.3 \mu$ s duration, and an eight-event deep derandomising buffer to permit sustained operation at trigger rates up to $10^{5}$ per second without deadtime. The custom SCT design, the ABCD, is implemented as a BiCMOS single chip application-specific- integrated-circuit (ASIC) in a radiation-tolerant technology [6]. Each ASIC has 128 channels with a common threshold for discrimination.

Following testing of the first generation $\mathrm{ABCD}$, the second generation, $\mathrm{ABCD} 2 \mathrm{~T}$, implemented a 4 bit DAC adjustment to the threshold at each channel to reduce excess threshold spread (trim DAC), and shielding the input pads for excess noise protection [7]. The third generation, ABCD3T, fixed poor matching in the circuit which generated the correction voltage for the discriminator threshold, and added a 2-bit range for the step of the trimDAC to cope with the deterioration in the trimming offset spread after irradiation [8].

\section{Barrel and endcap modules}

The SCT modules are made from four silicon microstrip sensors, glued in pairs on each side of a baseboard for total strip lengths of $12 \mathrm{~cm}$. The two over-lapping planes are slightly rotated with respect to each other by an angle of $40 \mathrm{mrad}$. This small stereo angle allows measurement of a space-point with coarse longitudinal resolution while retaining the high transverse spatial resolution with two nearly parallel planes. Each module is equipped with 12 ABCD chips on a hybrid, 6 on the top and 6 on the bottom side. In the barrel modules, the hybrid is placed near the centre of the module in a wrap-around configuration; in the endcap modules, the hybrid is placed at one end of the module and is double-sided. The different designs were driven principally by the difference in cylindrical and disk geometries. Photographs of the barrel and endcap modules are shown in Fig. 1 and Fig. 2.

Thermal management is critical to extract the heat of the ASICs ( 5 to $8 \mathrm{~W}$ ) and of the sensors, especially after the sensors are heavily radiation-damaged(1 to $2 \mathrm{~W}$ ). The baseboard

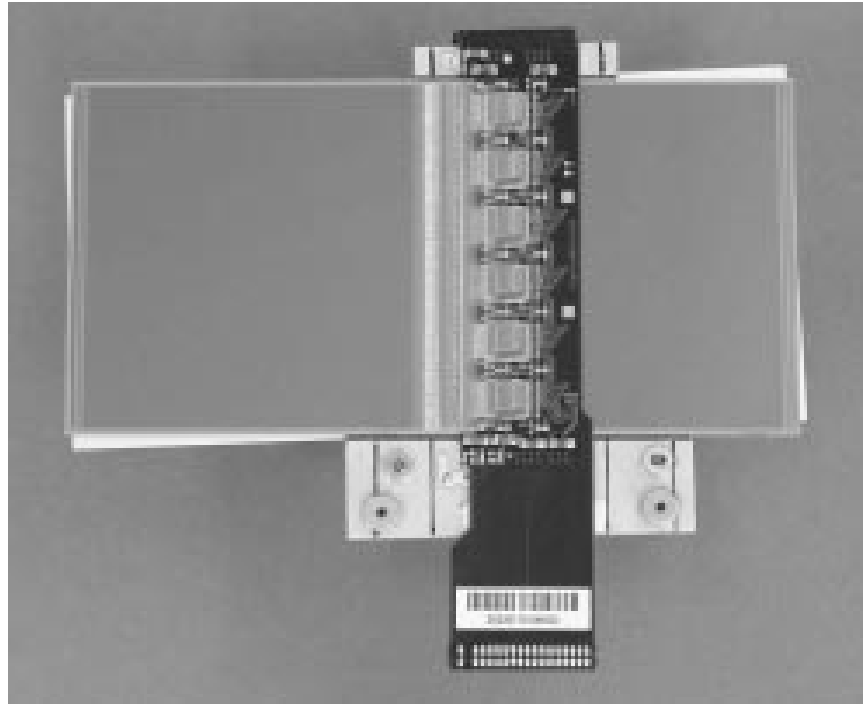

Fig. 1. ATLAS SCT barrel module

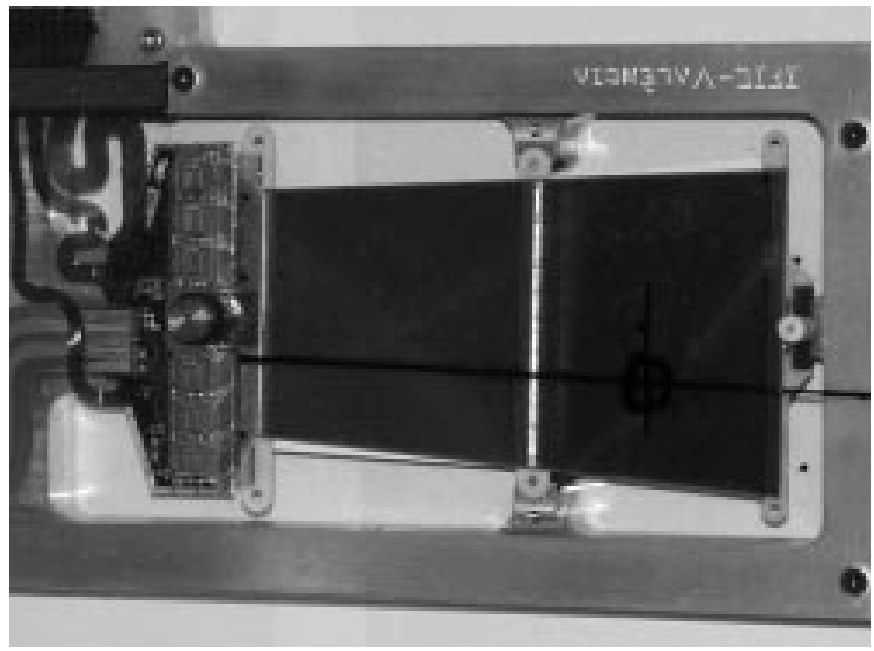

Fig. 2. ATLAS SCT endcap module, together with a module frame. A black line in the sensors was a line on a transparent protection plastic cover.

with a high thermal conductivity is a key element to prevent the thermal runaway of the sensors. The barrel module is cooled at one area at the wide tab, whereas the endcap module has two small cooling areas: one at the junction of the sensor and the hybrid, and the other at the far-end of the sensors.

Two modules, one barrel and one endcap, were irradiated prior to the beamtest to the fluence of $\sim 3 \times 10^{14}$ protons $/ \mathrm{cm}^{2}$ at the $24 \mathrm{GeV}$ proton synchrotron at CERN, equivalent to the $\sim 2 \times 10^{14} 1 \mathrm{MeV}$ neutrons $/ \mathrm{cm}^{2}$. The modules were irradiated cold and then annealed immediately after the irradiation for 7 days at $25{ }^{\circ} \mathrm{C}$ to simulate the effects of the warm-up for the maintenance in the real experiment. Additionally, the modules were at room temperature for several days during the beamtest 
preparation but otherwise kept cold $\left(<-10^{\circ} \mathrm{C}\right)$.

\section{BEAMTEST}

\section{A. Setup}

Two beamtests are reported here. In the first, three non-irradiated modules were tested: two barrel (k3103, k3104) and one endcap (FR-k81), all with ABCD2T chips. In the second, six modules were tested: two non-irradiated $(011,022)$ and one irradiated barrel (003), two non-irradiated (VAL-k3-165, CG-k3-170), and one irradiated endcap (VAL-k3-166) modules, all with ABCD3T chips [9]. Since the results of the second beamtest supersede and expand on the first, only those of the second test are presented in this paper. The characteristics of modules in the second test are summarized in Table II.

TABLE II

NAME, TYPE, ASIC, AND IRRADIATION CHARACTERISTICS OF THE MODULES IN THE SECOND BEAMTEST

\begin{tabular}{c|c|c|c|c}
\hline Beamtest id & Name & Type & ASIC & Irradiation \\
\hline $\bmod 1$ & 011 & barrel & ABCD3T & non-irrad \\
\hline $\bmod 2$ & 022 & barrel & ABCD3T & non-irrad \\
\hline $\bmod 3$ & 003 & barrel & ABCD3T & irrad \\
\hline $\bmod 4$ & VAL-k3-166 & endcap & ABCD3T & irrad \\
\hline $\operatorname{mod5}$ & VAL-k3-165 & endcap & ABCD3T & non-irrad \\
\hline $\bmod 6$ & CG-k3-170 & endcap & ABCD3T & non-irrad \\
\hline
\end{tabular}

The modules were attached to aluminium module frames and positioned in a thermo-box in the beamline. Three analogue readout "Si-telescopes" provided independent tracking with a position resolution of about $5 \mu \mathrm{m}$ in horizontal and in vertical directions. Pairs of modules, $\bmod 2$ and $\bmod 3$, and $\bmod 4$ and mod5, were sandwiched between telescope planes, all spaced at $30 \mathrm{~mm}$ intervals. The smearing by multiple scattering was estimated to be negligible by interpolating the positions of the incident particles with two adjacent telescopes. Readout was triggered by scintillators of $2 \mathrm{~cm} \times 2 \mathrm{~cm}$ placed upstream. The beam size (FWHM) was about $1 \mathrm{~cm} \mathrm{x} 1 \mathrm{~cm}$ in the modules.

The modules were cooled in two ways: the overall environment inside the thermo-box was cooled with a circulation of cold air of about $-20{ }^{\circ} \mathrm{C}$, and, in addition, the irradiated modules were cooled with a liquid cooling of about $-13{ }^{\circ} \mathrm{C}$. The temperatures of the barrel hybrid were about $0{ }^{\circ} \mathrm{C}$.

The temperatures of the sensors were estimated to be about $-10{ }^{\circ} \mathrm{C}$. Typical leakage currents of the non-irradiated and the irradiated modules were $0.2 \mu \mathrm{A}$ and $2 \mathrm{~mA}$, respectively. Problems were encountered in cooling the endcap modules, with their hybrids running at about $37{ }^{\circ} \mathrm{C}$ and $54{ }^{\circ} \mathrm{C}$ in the non-irradiated and the irradiated modules, respectively.

\section{$B$. Trim range setting in the irradiated modules}

After the irradiation, ABCD3T chips showed a problem in setting the trim DAC range of the trim circuitry. (This problem was fixed in the more recent ABCD3TA chips.) The ranges were stuck to 1 and 2 in $\bmod 3$, and 2 and 3 in $\bmod 4$, in the 4 chips illuminated by the beam. The number of channels masked due to this out-of-trimming was, however, small: 1 and 4 channels in the mod 3 and the mod 4 modules, respectively.

\section{Charge calibration}

The relations of the threshold voltages and the charges were calibrated, in situ, by injecting charges with the internal circuitry in the ABCD chips. The resulting calibration curves, averaged over the chips in the beam spot, are shown in Fig. 3.

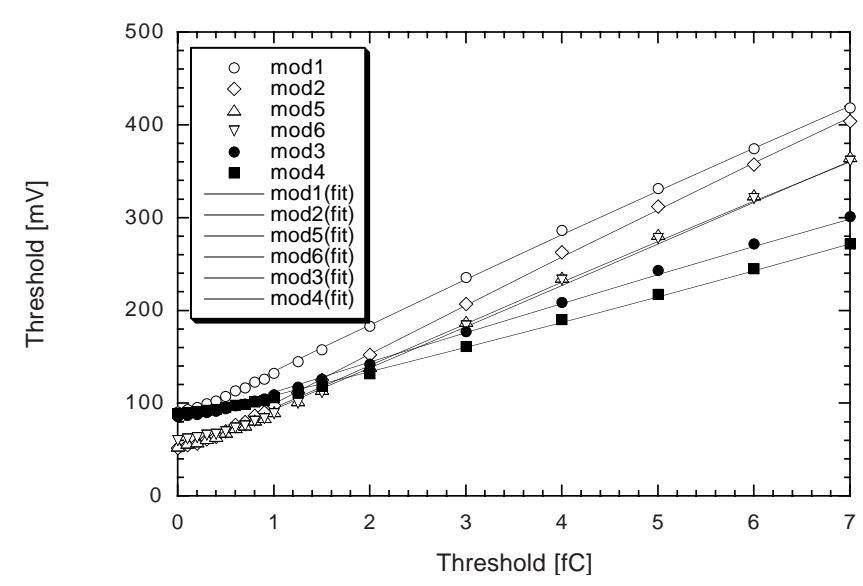

Fig. 3. Calibrations of the threshold in $\mathrm{mV}$ and $\mathrm{fC}$

The internal calibration circuitry required several corrections: the variation of the values of the charge injection capacitance (estimated to be $\sim 7 \%$ from test structures), and variation of charge scales due to chip temperatures $(0 \sim 5 \%)$ and radiation damage $(0 \sim 3 \%)$. These factors were determined separately for each module and applied in the analysis presented below.

\section{Data taking and analysis}

Two parameters were varied during data-taking: the sensor bias voltage, between 25 and $275 \mathrm{~V}$ for the non-irradiated and 150 and $500 \mathrm{~V}$ for the irradiated modules, and the threshold between 0.7 and $6 \mathrm{fC}$. In the following analyses, the conditions taken for a typical setting were, otherwise mentioned, (1) bias voltages of $150 \mathrm{~V}$ and $350 \mathrm{~V}$ for the non-irradiated and the irradiated modules, and (2) threshold at $1 \mathrm{fC}$. In the plots, the voltages of the irradiated modules were corrected for the voltage drops in the series resistance in the bias supply lines.

\section{DATA ANALYSIS}

\section{A. Pulse shape reconstruction}

The ABCD chip has, after amplification and shaping, discriminator circuitry. The output is sampled at a fixed phase of the $40 \mathrm{MHz}$ clock. Unlike at the LHC where the clock will be synchronised with collision frequency, in the beamtest the ar- 
rival time of the triggering particle is random relative to the clock phase. This interval was measured using a TDC which, when combined with the threshold scans, allowed the time distribution of the median pulse heights to be reconstructed.

The resulting time distributions of the median pulse heights are shown in Fig. 4 for the non-irradiated (average of mod1 and $\bmod 2)$ and for the irradiated $(\bmod 3)$ modules. In Fig. 4 , the offsets of the peaking were adjusted to be at $40 \mathrm{~ns}$. The impulse response curves of a first-order differential and third-order integration circuitry, CR-RC ${ }^{3}$, were fitted to the rising part of the pulses between 20 and $45 \mathrm{~ns}$. The peaking time of the non-irradiated module was thus found to be $22 \mathrm{~ns}$ and that of the irradiated $27 \mathrm{~ns}$. The peaking time of the non-irradiated chips is consistent with the ABCD specification. The peaking time of the irradiated module was longer due to a slower charge collection in the radiation-damaged sensors and a slower speed of the damaged amplifiers.

Another distinctive feature of the reconstructed pulse shapes was the shoulders seen after the peaking. This shoulder could not be reproduced in a simulation of the charge collection in the damaged sensors. Since this shoulder has not been observed in similar chips without the discriminators [10], it could be a physical feedback from the discriminator.

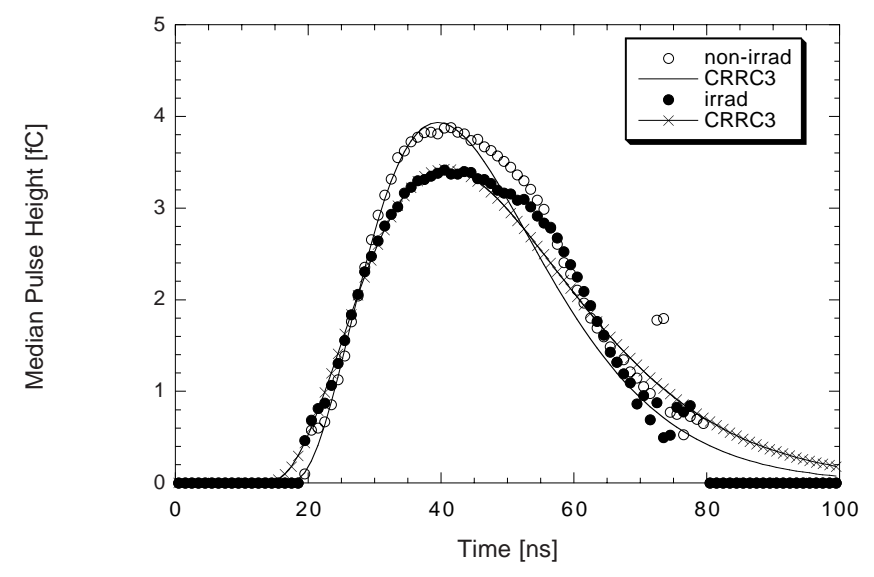

Fig. 4. Median pulse height distributions as a function of time between trigger and clock phase: non-irradiated (open circle) and irradiated (filled circle) modules, and the impulse responses of CR-RC ${ }^{3}$ circuitry with the peaking times of 22 and $27 \mathrm{~ns}$, respectively

The peaking times of the modules as a function of bias voltages were shown in Fig. 5. The error bars are statistical only, from the $\mathrm{CR}-\mathrm{RC}^{3}$ fits to the average pulse shapes. In the non-irradiated modules, the pulses slowed down slightly, by $3 \mathrm{~ns}$, below $150 \mathrm{~V}$ to $50 \mathrm{~V}$. The endcap modules were slower than the barrel modules, by $5 \mathrm{~ns}$ in the non-irradiated and $7 \mathrm{~ns}$ in the irradiated modules. This deterioration might be caused by the higher temperature of the chips in the endcap hybrids.

In the following analyses, events have been selected in which time was in the peak region between 35 and $45 \mathrm{~ns}$. No correction was made to the range in other bias voltages since the move of the peak position was small.

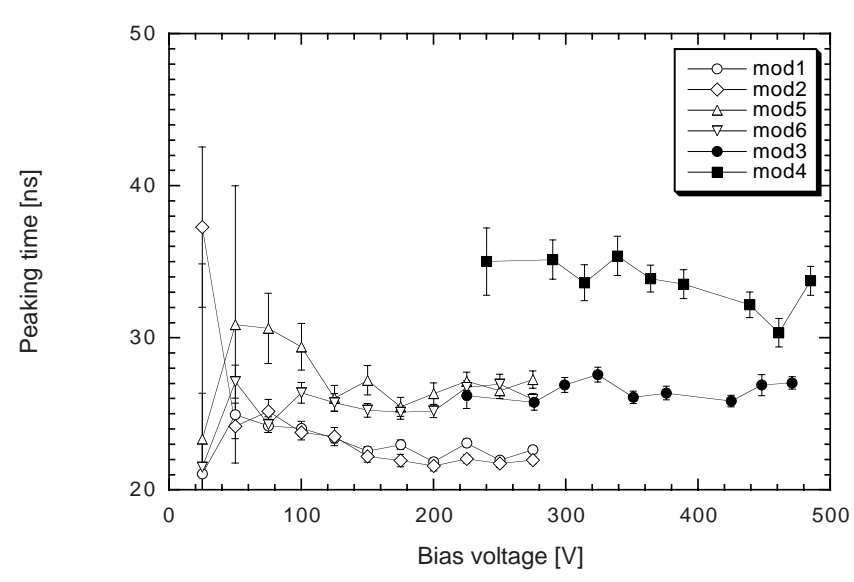

Fig. 5. Bias voltage dependence of the peaking times

\section{B. Position resolutions}

Consecutive hit-strips were clusterized within each sensing plane to define a hit-cluster with the geometrical centre as the position of the "cluster centre" and the number of strips as the "cluster width". The trajectories of the incident particles were defined by the two adjacent telescopes in order to minimize the effect of multiple scattering.

The differences of the cluster centres from the projected hit gave the position resolution of the sensing plane. The deviation distributions of one module, mod1, is shown in Fig. 6, for all events (circle) and for the cluster width greater than 1 , the "multi-hits", (diamond). Also shown are the Gaussian functions fitted without error-weighting.

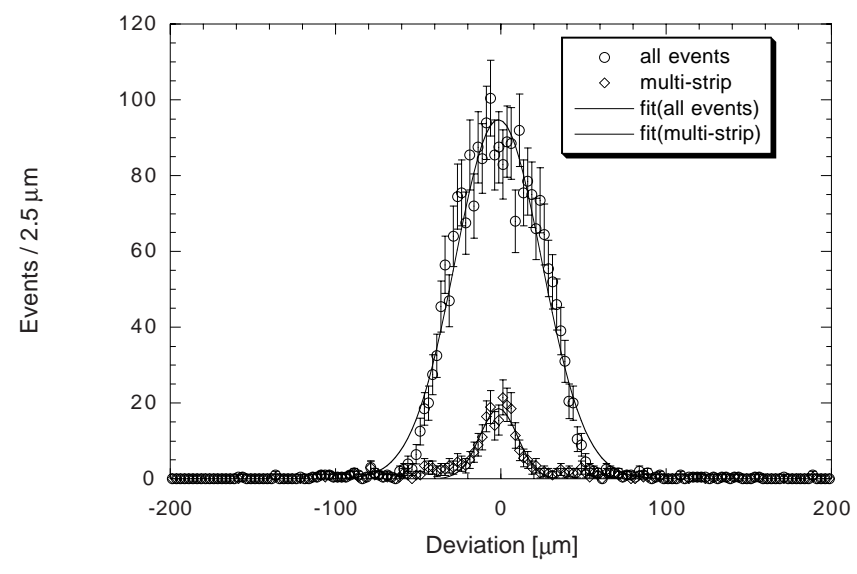

Fig. 6. Deviations of the cluster centre from the expected positions of incident particles of the mod 1 module: all events (circles) and the events with multi-hits (diamonds), with Gaussian fits without weighting

The distribution of all events deviates from Gaussian, is 
closer to a uniform (box) distribution as expected for discriminated binary readout with a strip pitch of $80 \mu \mathrm{m}$ but affected by the telescope resolution and noise hits. The distribution of the multi-hits is much narrower due to the predominance of two-strip hits where the tracks pass through a small inter-strip region (two-strip-hit sensitive region).

The root-mean-square (RMS) resolution of the distribution, being less affected by the telescope resolution and the noise hits, was obtained by dividing the Gaussian sigmas by a factor 1.24 which is the ratio of the RMS and the Gaussian sigma of a uniform distribution. The resulting RMS resolutions were $22.5 \mu \mathrm{m}$ and $10 \mu \mathrm{m}$ for the all events and multi-hits events, respectively.

In analysing the endcap modules, the variable pitch of fan geometry was corrected to the average strip pitch of $86 \mu \mathrm{m}$ at the beam spot. The RMS resolutions are shown in Fig. 7 and Fig. 8 as a function of bias voltage and threshold. Those of the modules outside the telescopes were corrected for the multiple scattering effect. The RMS resolutions of the barrel and the endcap modules were about $23 \mu \mathrm{m}$ and $26 \mu \mathrm{m}$, consistent with their pitch of $80 \mu \mathrm{m}$ and $86 \mu \mathrm{m}$, respectively, although those of the endcap modules were slightly worse than the expected.

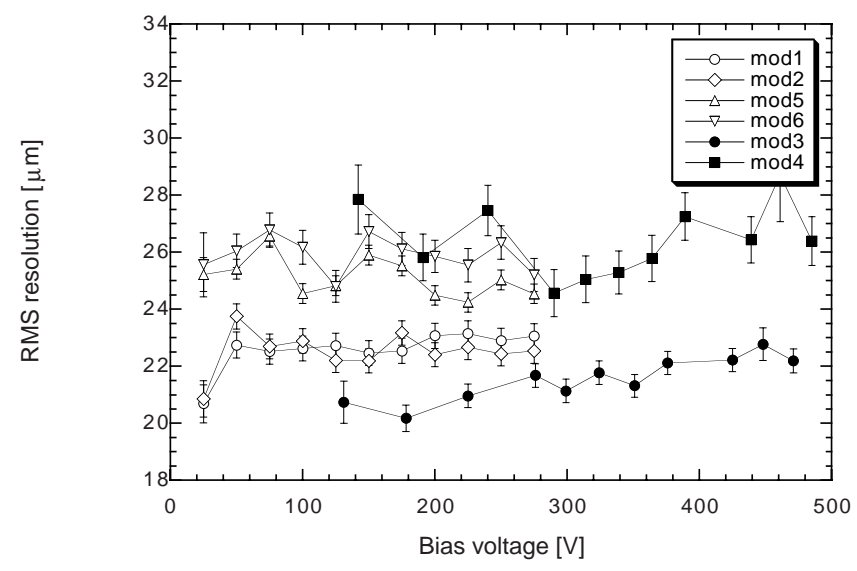

Fig. 7. RMS resolutions as a function of bias voltage.

\section{Mean cluster width}

The fractions of the multi-hits, parameterized as the mean cluster width, are shown in Fig. 9 and in Fig. 10 as a function of bias voltage and threshold, respectively. There seems to be a correlation between the decrease in the RMS resolutions and the increase in the fraction of multi-hits, e.g., below $2 \mathrm{fC}$ in Fig. 8 and Fig. 10. The better resolution of the multi-hit events can be seen in Fig. 6.

\section{Efficiency scans and Median charges}

By counting hits with cluster centres within a window of the incident particles (full width of $500 \mu \mathrm{m}$ in the analysis), the ef-

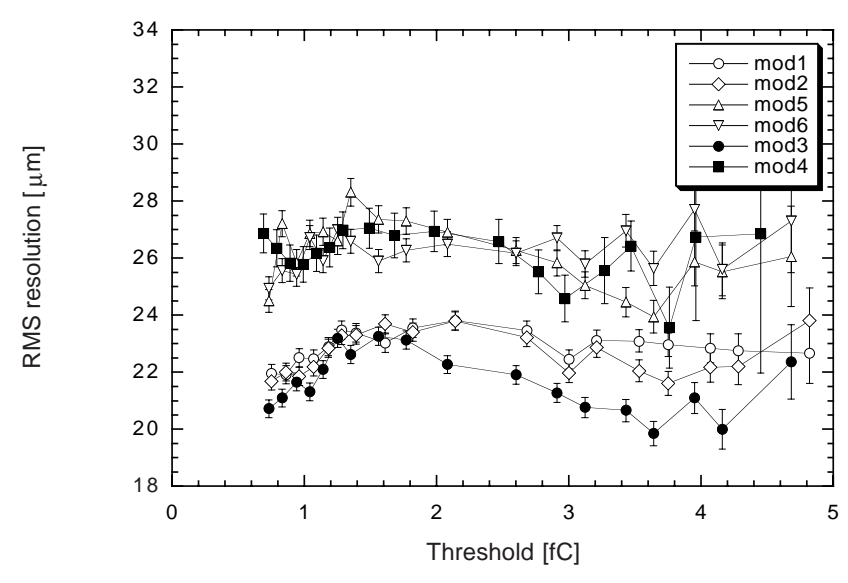

Fig. 8. RMS resolutions as a function of threshold.

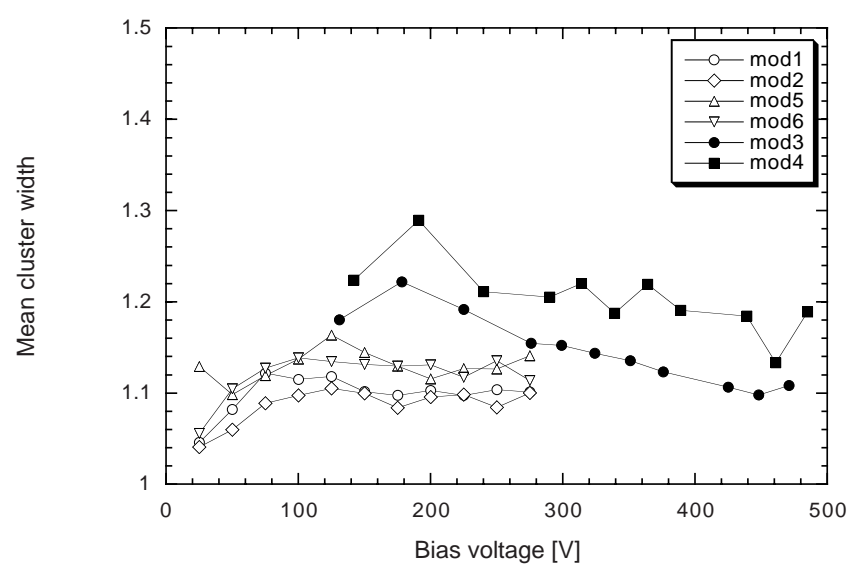

Fig. 9. Mean cluster widths as a function of bias voltage

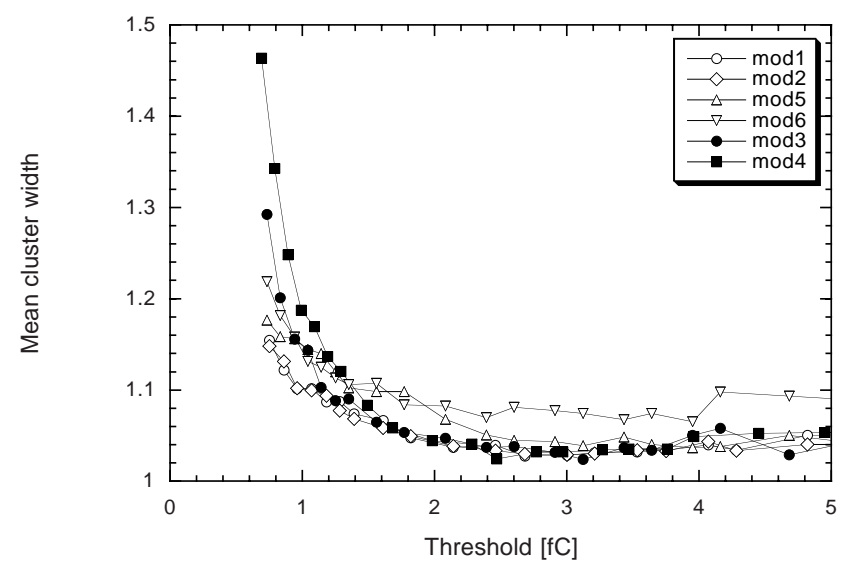

Fig. 10. Mean cluster widths as a function of threshold

ficiency at a threshold can be obtained. The pulse height (Landau) distribution is reconstructed as an efficiency curve by scanning the threshold. An example is shown in Fig. 11 for 
the irradiated module, $\bmod 3$, where with the bias voltage is also scanned. The threshold of the $50 \%$ efficiency is the median charge of the pulse height (Landau) distribution.

In order to obtain the threshold of $50 \%$ efficiency, a modified error function, (1), was fitted to the efficiency scans,

$$
\operatorname{eff}(q)=p_{3}(1-\operatorname{erf}(T \cdot f(T)))
$$

where

$$
\begin{gathered}
f(T)=1+0.6 \cdot \tanh \left(-p_{4} \cdot T\right) \text { and } \\
T=\left(q-p_{1}\right) /\left(\sqrt{2} \cdot p_{2}\right) .
\end{gathered}
$$

The function, erf, was the integral of the Gaussian distribution. The function, $f(T)$, is an empirical function to modify the Gaussian to the Gaussian-convoluted Landau distribution. The fitting parameters expressed the median $\left(\mathrm{p}_{1}\right)$, the width $\left(\mathrm{p}_{2}\right)$, the saturation $\left(\mathrm{p}_{3}\right)$, and the skew $\left(\mathrm{p}_{4}\right)$.

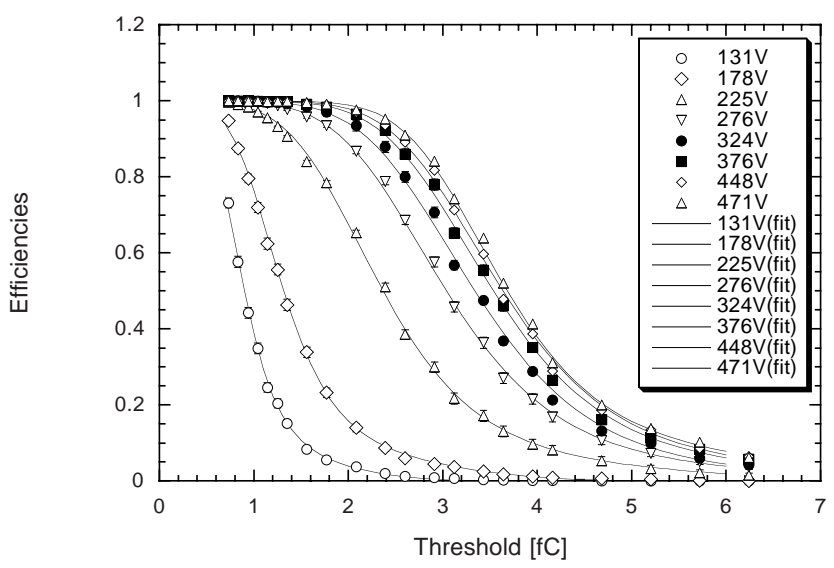

Fig. 11. Threshold scan of the efficiencies at various bias voltages in the irradiated barrel module, mod3. The lines are fits to the empirical formula in the text

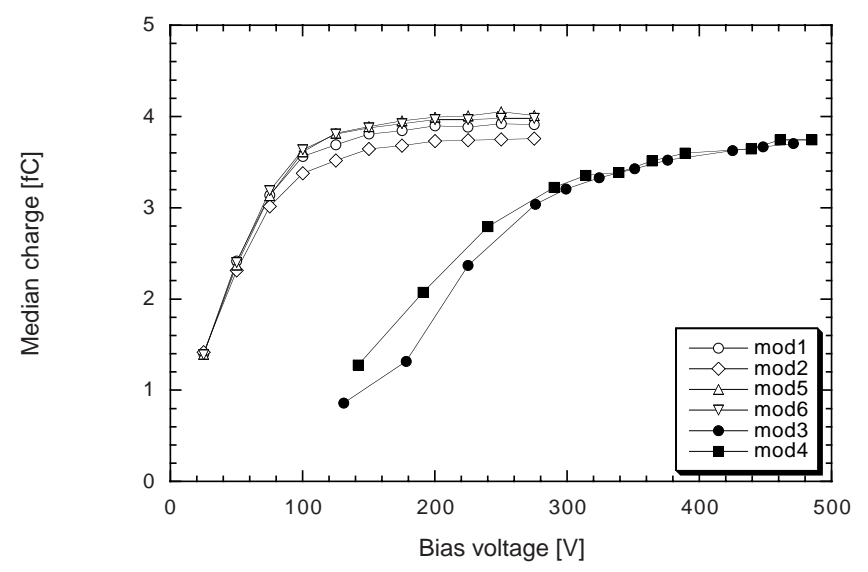

Fig. 12. Bias voltage dependence of the median charges.

The median charges of the modules obtained are shown in
Fig. 12 as a function of the bias voltage. The median charges of the non-irradiated modules saturate above $150 \mathrm{~V}$ to the charges between $3.8 \mathrm{fC}$ and $4.0 \mathrm{fC}$. The saturations of the median charges of the irradiated modules were slower, reaching around $3.8 \mathrm{fC}$ at $500 \mathrm{~V}$. The full depletion voltages of the non-irradiated and irradiated sensors were about $70 \mathrm{~V}$ and $300 \mathrm{~V}$, respectively.

Usually the charge collection of the irradiated silicon sensors does not reach that of non-irradiated ones [11]. The correction of the charge scale due to radiation damage could be insufficient. The median charges of the two non-irradiated barrel modules do not coincide, nor do those of the barrel and the endcap modules. These differences could be attributed to the chip-by-chip variation of the calibration capacitance and insufficient corrections on calibration scales due to temperature.

\section{E. Noise occupancies and ENC}

Hits which were out of time (time $<15 \mathrm{~ns}$ ) and out of track window (twice the efficiency window) were counted as noise hits due to electronics noise. The resulting number of hit strips was divided by the number of strips outside the track window to obtain the noise occupancy. The noise occupancies of the modules are shown in Fig. 13 as a function of threshold-squared.

The noise occupancies at $1 \mathrm{fC}$ of both the non-irradiated barrel and endcap modules were well below, and the irradiated barrel module was about satisfying the requirement for the ATLAS SCT modules: $5 \times 10^{-4}$ [1]. Those of the endcap modules were an order larger than of the barrel modules which could be attributed to the difference in chip temperatures.

The relation between the noise occupancy and the threshold-squared $\left(\mathrm{th}^{2}\right)$ is a straight line in logarithmic scale of occupancy because the occupancy can be approximated as

$$
\text { occupancy }(\text { th }) \propto \exp \left(-(1 / 2)\left((\text { th })^{2} / \sigma^{2}\right)\right)
$$

where $\sigma$ is the equivalent-noise-charge (ENC) of the amplifier [12]. The ENCs (occuENC) were fitted in $t^{2}$ below $3 \mathrm{fC}^{2}$, in general, at low thresholds. The occuENC had little bias voltage dependence. The average above $100 \mathrm{~V}$ (non-irrad) and $300 \mathrm{~V}$ (irrad) are summarized in Table III. The ENCs (calibENC) were obtained separately in the in-situ calibration, with an average of ENCs at 1,2, and 3fC, including the corrections in the section III.C. In comparison, calibENC's obtained at high thresholds were systematically larger than occuENC's obtained at low thresholds.

\section{F. Signal-to-Noise ratios}

One way of cancelling the uncertainty of the calibration capacitance is to take the ratio of the median charges to the ENCs, the signal-to-noise ratios $(\mathrm{S} / \mathrm{N})$. The $\mathrm{S} / \mathrm{N}$ ratio is also an indicator of the performance since a $\mathrm{S} / \mathrm{N}>9$ is required in practical use from experience. In order to calculate the $\mathrm{S} / \mathrm{N}$, the worse ENCs, calibENC, were used to be conservative. The resulting $\mathrm{S} / \mathrm{N}$ ratios are shown in Fig. 14 as a function of bias 


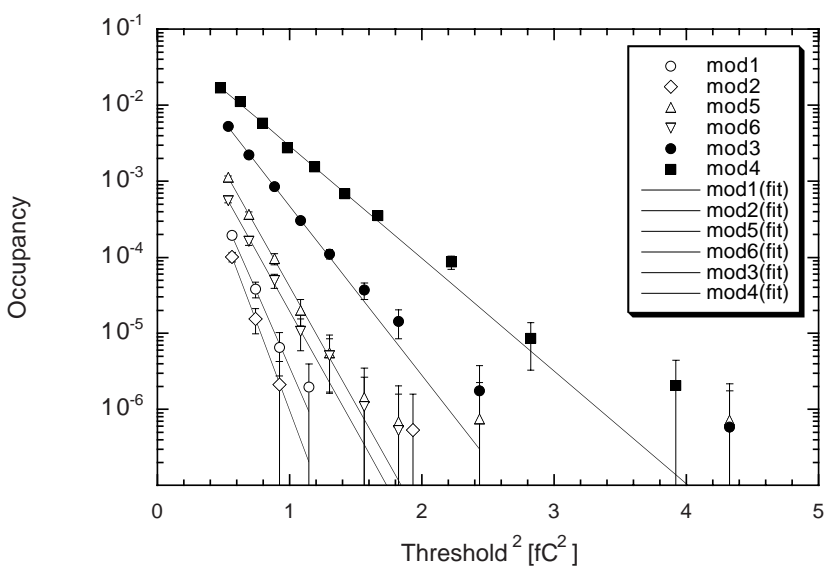

Fig. 13. Noise occupancies as a function of (threshold) ${ }^{2}$. The lines are fits to the function, $\exp \left(-1 / 2 *(\mathrm{th} / \sigma)^{2}\right)$

TABLE III

COMPARISON OF ENCS OBTAINED FROM THE OCCUPANCY AND THE IN-SITU CALIBRATION

\begin{tabular}{c|c|c|c}
\hline mod & occuENC[fC] & calibENC[fC] & Ratio \\
\hline 1 & 0.239 & 0.245 & 0.976 \\
\hline 2 & 0.222 & 0.234 & 0.949 \\
\hline 3 & 0.311 & 0.342 & 0.910 \\
\hline 4 & 0.385 & 0.411 & 0.937 \\
\hline 5 & 0.264 & 0.291 & 0.907 \\
\hline 6 & 0.264 & 0.290 & 0.910 \\
\hline
\end{tabular}

voltage.

A good match of the $\mathrm{S} / \mathrm{N}$ was seen between two barrel and two endcap modules, which supported the differences of the median charges were caused by the chip-to-chip variation of the calibration capacitance. The $\mathrm{S} / \mathrm{N}>16$ was reached above $150 \mathrm{~V}$ in the non-irradiated barrel, and the $\mathrm{S} / \mathrm{N}>10$ above $300 \mathrm{~V}$ in the irradiated barrel modules. The $\mathrm{S} / \mathrm{N}$ of endcap modules were considerably lower than those of barrel modules, which can be attributed to the higher chip temperatures.

\section{G. Charge collection in the interstrip regions}

Since the charges moving in the midway region between two strips generate currents in both strips, the induced charge on either strip is halved. This is seen in Fig. 15 where "eta" is the normalized distance from the strip centres. Although degradation was seen in high thresholds, the efficiencies were still high at $1 \mathrm{fC}$, the expected operating threshold. The ratios of the median charges in the strip and the inter-strip region were about 0.95 and 0.85 in the non-irradiated and the irradiated modules. The thresholds where the loss of efficiency starts could be estimated by applying these factors to the efficiency curves, e.g., in Fig. 11.

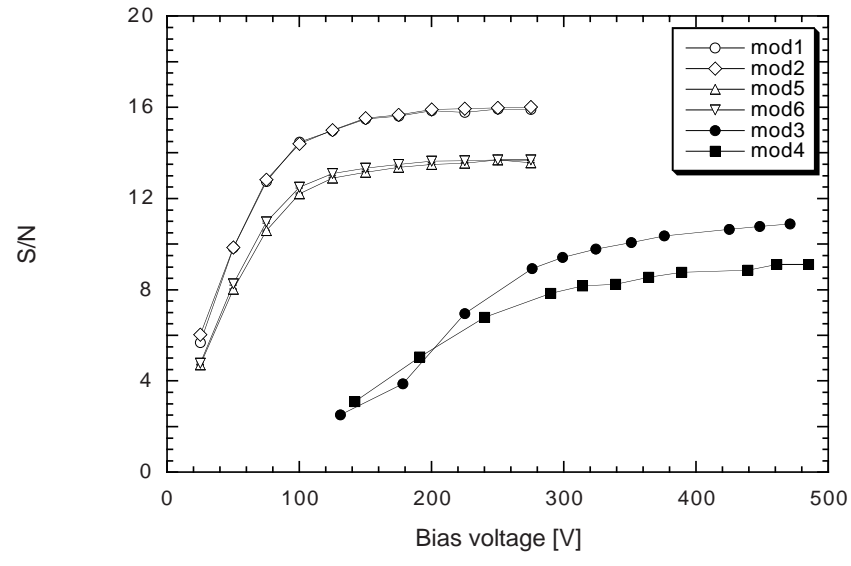

Fig. 14. Signal-to-noise ratios as a function of bias voltage. The noises were averages of 1,2 , and $3 \mathrm{fC}$ of the in-situ calibration

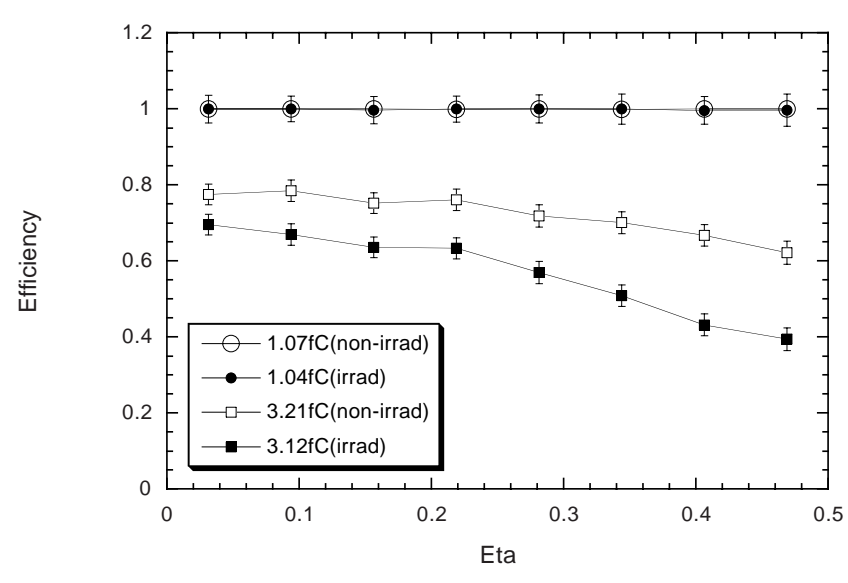

Fig. 15. Efficiencies between the strips. "Eta" defines the distance from the strips: 0 at strip and 0.5 at midway.

\section{H. Common-mode noise}

In the case the electronics system has external noise pick-up or an internal oscillation, excess hits appear across many strips as common-mode noise. The distributions of number of hits in the non-irradiated (average of mod 1 and mod2) and irradiated $(\bmod 3)$ modules are shown in Fig. 16 at the threshold of $0.7 \mathrm{fC}$. No excess common-mode noise was observed. The distribution of the irradiated module is the Poisson distribution with a mean occupancy consistent with the threshold, 768 strips (per side), and 3 time bins.

\section{SUMMARY}

Two beamtests were carried out by using $4 \mathrm{GeV} / \mathrm{c}$ pions from the $12 \mathrm{GeV}$ proton synchrotron at KEK for the ATLAS SCT barrel and endcap modules fully equipped with the SCT specification components, including modules irradiated to a fluence of $\sim 3 \times 10^{14}$ protons $/ \mathrm{cm}^{2}$.

A pulse shape analysis confirmed the peaking time of the 


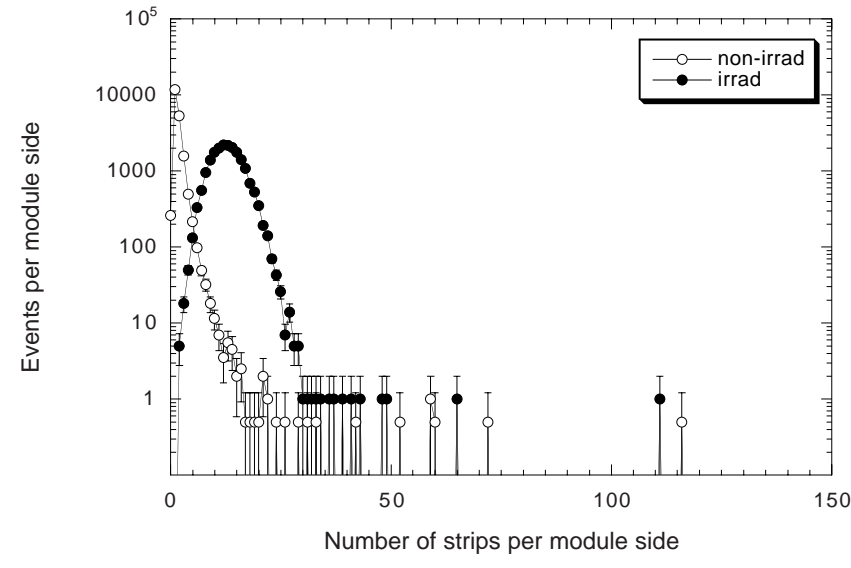

Fig. 16. Distribution of the number of strips hit in the non-irradiated (average of $\bmod 1$ and $\bmod 2)$ and irradiated $(\bmod 3)$ modules at $0.7 \mathrm{fC}$ thresholds

amplifier to be $22 \mathrm{~ns}$ in the non-irradiated modules with deteriorated to $27 \mathrm{~ns}$ in the irradiated modules. The RMS position resolutions were consistent with the uniform distribution of the pitch of $80 \mu \mathrm{m}$ in the barrel and of $86 \mu \mathrm{m}$ in the endcap at the beam spot. The variations of the resolutions were correlated to the fraction of the multi-hits events.

The median charges of the pulse height (Landau) distribution were obtained from discriminator threshold scans, and saturated around $3.8 \mathrm{fC}$ in the non-irradiated $(>150 \mathrm{~V})$ and irradiated $(500 \mathrm{~V})$ modules. The noise occupancies of the non-irradiated barrel and endcap and the irradiated barrel module satisfied the ATLAS SCT requirement: $5 \times 10^{-4}$.

By using the median charges and the ENCs of in-situ calibration, the signal-to-noise ratios were obtained to be $>16$ in the non-irradiated $(>150 \mathrm{~V})$ and $>10$ in the irradiated $(>300 \mathrm{~V})$ barrel modules. The matching of the $\mathrm{S} / \mathrm{N}$ of the non-irradiated modules indicated the spread of the median charges could be caused by the variation of the chip-by-chip calibration capacitance.

The endcap modules were worse in two respects: rise times of the pulses, and noises. These could be attributed to higher temperature of the readout ASICs and improvement is foreseen. No excess common-mode was observed in the beamtests.

\section{ACKNOWLEDGEMENT}

The authors wish to acknowledge the beam channel crews of the $\pi 2$ beamline of the KEK PS, L. Sospedra of Univ. of Valencia for beamtest preparation, P. Kodys of Charles Univ. for data analysis, and all the support from the ATLAS SCT collaboration.

\section{REFERENCES}

[1] ATLAS Inner Detector Technical Design Report, CERN/LHCC/97-17, ATLAS TDR 5, 30 April 1997

[2] Y. Unno, "ATLAS silicon microstrip Semiconductor Tracker (SCT), Nucl. Instr. Meth., Vol. A453, pp.109-120, 2000

[3] Y. Unno, T. Yamashita, S. Terada, T. Kohriki, G. Moorhead, Y. Iwata et al., "Evaluation of Radiation Damaged P-in-n and N-in-n Silicon Microstrip Detectors", IEEE Trans. Nucl. Sci., Vol. 46, pp. 1957-1963, 1999

[4] Y. Unno, (1) Testbeam experiment T450, KEK, 10-20 Dec. 1999, (2) Testbeam experiment T478, KEK, 28 Nov.-10 Dec. 2000

[5] Sensors fabricated by Hamamatsu Photonics, 1126-1, Ichino-cho, Hamamatsu-shi 435, Japan

[6] DMILL technology, TEMIC Semiconductors, La Chantrerie, F-44306 Nantes, France

[7] F. Anghinolfi, A. Ciocio, A. Clark, W. Dabrowski, Z. Dolezal, D. Dorfan et al., "Performance of the Electrical Module Prototypes for the ATLAS Silicon Tracker", Snowmass 1999, Electronics for LHC experiments, pp. 118-122

[8] W. Dabrowski, F. Anghinolfi, C. Buttar, V. Cindro, A.G. Clark, I. Dawson et al., "Design and Performance of the ABCD Chip for the Binary Readout of Silicon Strip Detectors in the ATLAS Semiconductor Tracker", IEEE Trans. Nucl. Sci. Vol. 47, pp. 1843-1850, 2000

[9] The FR-, VAL-, and CG-modules were prepared by Univ. Freiburg, Univ. Valencia, and CERN-Univ. Geneva, respectively. The rest of the modules were prepared by KEK

[10] W. Dabrowski, private communication

[11] T. Akimoto, S. Arai, K. Hara, T. Nakayama, Y. Ikegami, Y. Iwata et al., "Characteristics of irradiated silicon microstrip detectors with $\langle 100\rangle$ and $<111>$ substrates", Nucl. Instr. Meth., Vol. A466, pp. 354-358, 2001

[12] T. Dubbs, S. Kashigin, M. Kratzer, W. Kroeger, T. Pulliam, H.F.W. Sadrozinski et al., "Noise Determination in Silicon Micro Strips", IEEE Trans. Nucl. Sci. Vol. 43, pp. 1119-1122, 1996 